Series A

I. MATHEMATICA

379

\title{
ZUR THEORIE LINEARER \\ DIFFERENTIALGLEICHUNGEN IM KOMPLEXEN
}

VON

H. WITTICH

Rolf Nevandinna zum 70 Geburtstag

HELSINKI 1966

SUOMALAINEN TIEDEAKATEMIA

https://doi.org/10.5186/aasfm.1966.379 
Am 10. September 1965 vorgelegt von P. J. Mrrberg und Oldi Lehto 


\section{Zur Theorie linearer Differentialgleichungen im Komplexen}

Nach dem Existenzsatz von A. L. Cauchy sind bei passenden Annahmen die Lösungen gewöhnlicher Differentialgleichungen im Komplexen analytische Funktionen einer komplexen Veränderlichen. Die Untersuchung der Lösungen stützt sich auf Sätze der Funktionentheorie. Umgekehrt ermöglichen funktionentheoretische Hilfsmittel die Bestimmung von Klassen gewöhnlicher Differentialgleichungen, deren Lösungen vorgeschriebene Eigenschaften aufweisen. Eine Frage in dieser Richtung behandelten schon 1856 Ch. Briot und J. C. Bouquet [2] mit der Aufgabe, die eindeutigen Lösungen der Differentialgleichung $P\left(w, w^{\prime}\right)=0 \quad$ zu bestimmen, wo $P\left(w, w^{\prime}\right)$ ein Polynom in $w$ und $w^{\prime}$ ist. In diesem Zusammenhang ist noch eine Untersuchung von J. Malmquist [8] zu erwähnen. Danach ist für rationales $R(z, w)$ entweder $w^{\prime}=R(z, w)$ eine Riccatische Differentialgleichung, oder jede im grossen eindeutige Lösung $w(z)$ von $w^{\prime}=R(z, w)$ ist eine rationale Funktion.

Untersuchungen von L. Fuchs [5], die durch B. Riemanns Abhandlung über die hypergeometrische Differentialgleichung angeregt wurden, verfolgen das Ziel, Zusammenhänge zwischen dem Anwachsen der Lösungen linearer Differentialgleichungen und ihrer Koeffizienten aufzudecken, was für Stellen der Bestimmtheit vollständig gelungen ist. Es liegt nun nahe, auch Stellen der Unbestimmtheit zu betrachten. Das soll im folgenden geschehen, und zwar für lineare Differentialgleichungen

$$
w^{(n)}+a_{n-1}(z) w^{(n-1)}+\ldots+a_{o}(z) w=0, \quad a_{o}(z) \neq 0,
$$

mit ganzen Koeffizienten und für lineare Differentialgleichungen zweiter Ordnung der Form

$$
z^{2} w^{\prime \prime}+z a(z) w^{\prime}+b(z) w=0
$$

mit Koeffizienten $a(z)$ und $b(z)$, die in einer Umgebung von $z=\infty$ holomorph sind. Eine eingehendere Betrachtung der speziellen Gleichungen $\left(D^{\prime}\right)$ ist deshalb gerechtfertigt, weil die gewonnenen Resultate zur Kennzeichnung von solchen Differentialgleichungen zweiter Ordnung führen, die in der mathematischen Physik seit langer Zeit eine Rolle spielen. Diese Differentialgleichungen ergeben sich, wenn man, grob gesprochen, ein 
möglichst langsames Anwachsen der zu einer Stelle der Unbestimmtheit gehörigen kanonischen Lösungen fordert.

Ein der zu behandelnden Aufgabe angemessenes Hilfsmittel ist die Theorie der Wertverteilung*), wie sie rom Jubilar in ihren wesentlichen Teilen entwickelt wurde.

1. In der Differentialgleichung (D) seiən die Koeffizienten ganze Funktionen. Dann ist jede Lösung $w(z)$ von (D) eine ganze Funktion. Wird (D) als System

$$
\begin{array}{r}
\frac{d w_{j}}{d z}=f_{j}\left(z, w_{1}, \ldots, w_{n}\right), \quad w_{1}=w \text { und } \frac{d w_{j}}{d z}=w_{j+1}, \\
j=1, \ldots, n-1,
\end{array}
$$

geschricken, dam ergibt sich nach der Majorantenmethode von $A$. L. ('auchy: Sind alle Koeffizienten $a_{j}(z)$ Polynome $a_{j}(z)=A_{j} z^{\alpha_{j}}+\ldots$, dann ist jede Löstiog von (D) von endlicher Wachstumsordnung.

Diese Aussage lässt sich auch auf folgende Art beweisen. Es sei $y=\left(w_{1}, \ldots, w_{n}\right)$ und $\|y\|=\left(\sum_{1}^{n} \mid w_{j}\right)^{2}$.

Bei festem $\varphi, z=r e^{i \varphi}$, ist $\|y\|$ eine Funktion von $r$, für die wegen des Eindeutigkeitssatzes $\|y\| \neq 0$ gilt. Weiter ist $\frac{d}{d r} \| y \leqq \frac{d y}{d z} \cdot$ Mit $\alpha=\max \alpha_{j}$ gilt für alle $r \geqq r_{o}>1 \quad\left|a_{j}(z)\right| \leqq c r^{\alpha}$. Aus $\left|w_{j}^{\prime}\right|=\left|w_{j+1}\right|$, $j=1, \ldots, n-1$ und $\left|u_{n}^{\prime} \leqq \sum_{j=1}^{n-1}\right| a_{j}(z)\left|w_{j}(z)\right|$ folgt

$$
\sum_{1}^{n}\left|w_{j}^{\prime}\right|^{2} \leqq\|y\|^{2}+c_{1} r^{2 x}\|y\|^{2} \leqq c_{2} r^{2 \alpha}\|y\|^{2}
$$

also

$$
\frac{d}{d r}\|y\| \leqq \frac{d y}{d z} \mid \leqq c_{3} r^{\alpha}\|y\| \text { und } \log \|y\| \leqq c_{4} r^{\alpha+1} .
$$

Die letzte Abschätzung gilt für alle $z$ auf $|z|=r$, also auch dort, wo $|w(\zeta)|=M(r, w)=\max _{z ;=r}|w(z)|$ ist. Aus $\log M(r, w) \leqq \log \|y\| \leqq c_{4} r^{\alpha+1}$ folgt die Behauptung

$$
\lambda(w)=\varlimsup_{r \rightarrow \infty} \frac{\log \log M(r, w)}{\log r} \leqq \alpha+1<\infty .
$$

Schärfere Abschätzungen für die Ordnungen $\lambda(w)$ der Lösungen $w(z)$ gewinnt man mit der Methode des Zentralindex bei ganzen transzendenten Funktionen ([12] und [13]).

*) Man vgl. dazu [7], [9] und [13]. 
Es sei nun $w_{1}(z), \ldots, w_{n}(z)$ ein Fundamentalsystem ron (D) mit der Eigenschaft $\lambda\left(w_{j}\right)<\infty$ für $j=1,2, \ldots, n$. Die mit dem Fundamentalsystem gebildete Wronskische Determinante $W\left(w_{1}, \ldots, w_{n}\right)=W(z)$ ist eine ganze Funktion von endlicher Ordnung. Wegen $a_{n-1}(z)=-\frac{W^{\prime}(z)}{W(z)}$ gilt für die Schmiegungsfunktion von $a_{n-1}(z) \quad m\left(r, a_{n-1}(z)\right)=O(\log r)$, d.h. $a_{n-1}(z)$ ist ein Polynom. Damit folgt für $n=2$ wegen $a_{0}(z)=-\frac{w^{\prime \prime}}{w}-a_{1}(z) \frac{w^{\prime}}{w}$ durch mehrfache Anwendung des Satzes über die Schmiegungsfunktion der logarithmischen Ableitung $m\left(r, a_{0}(z)\right)=$ $O(\log r): \quad a_{0}(z)$ ist ebenfalls ein Polynom. Zum Beweis der analogen Aussage im Fall $n>2$ werden ähnliche Betrachtungen mit einer Reduktionsmethode von L. Fuchs kombiniert.

In der Differentialgleichung

$$
L_{m}(y)=y^{(m)}+A_{m-1}(z) y^{(m-1)}+\ldots+A_{0}(z) y=0
$$

seien die Koeffizienten $A_{j}(z)$ in $|z|<\infty$ analytische Funktionen. (1) besitze ein Fundamentalsystem $y_{1}(z), \ldots, y_{m}(z)$ von Lösungen, die in $|z|<\infty$ meromorph und von endlicher Ordnung sein sollen. Mit $v(\tilde{z})=$ $\frac{d}{d z}\left(\frac{y(z)}{y_{1}(z)}\right)$ ergibt sich für $v(z)$ die Differentialgleichung

$$
L_{m-1}(v)=v^{(m-1)}+B_{m-2}(z) v^{(m-2)}+\ldots+B_{o}(z) v=0 .
$$

Dabei ist für $j=0,1, \ldots, m-2$ und mit $A_{m}(z)=1$

$$
B_{j}(z)=A_{j+1}(z)+\sum_{i=1}^{m-j-1} c_{j r} A_{j+1+v}(z) \frac{y_{1}^{(v)}}{y_{1}} ;
$$

die Konstanten $c_{j}$, sind gewisse Binomialkoeffizienten.

Aus (2) folgt, dass die in $|z|<\infty$ meromorphen Funktionen $B_{j}(z)$ von endlicher Ordnung sind. Es ist unmittelbar zu sehen, dass die Lösungen $v_{j}(z)=\frac{d}{d z} \frac{y_{j+1}(z)}{y_{1}(z)}, \quad j=1, \ldots, m-1, \quad$ ein Fundamentalsystem von $\left(1^{\prime}\right)$ bilden, wobei noch $\lambda\left(v_{j}\right)<\infty$ gilt, wenn $\lambda\left(v_{j}\right)=\varlimsup_{r \rightarrow \infty} \frac{\log T\left(r, v_{j}\right)}{\log r}$ die Ordnung von $v_{j}(z)$ ist. Nun gilt der

Hilfssatz: Aus $\mathrm{m}\left(r, B_{j}(z)\right)=O(\log r), j=0,1, \ldots, m-2$, folgt $m\left(r, A_{i}(z)\right)=O(\log r), \quad i=0,1, \ldots, m-1$.

Beweis: Aus $B_{m-2}(z)=A_{m-1}(z)+c_{m-2,1} \frac{y_{1}^{\prime}}{y_{1}}$ folgt $m\left(r, A_{m-1}(z)\right) \leqq$ $m\left(r, B_{m-2}\right)+m\left(r, \frac{y_{1}^{\prime}}{y_{1}}\right)+O(1) \leqq c \log r$, also die Behauptung für $i=m-1$. 
Damit ergibt sich auf ähnliche Weise $m\left(r, A_{m-2}\right)=O(\log r)$ u.s.w. bis $m\left(r, A_{1}\right)=O(\log r)$ und schliesslich $m\left(r, A_{0}(z)\right)=O(\log r)$ aus

$$
A_{0}(z)=-\frac{y^{(m)}}{y}-A_{m-1}(z) \frac{y^{(m-1)}}{y}-\ldots-A_{1}(z) \frac{y^{\prime}}{y} .
$$

Nun wird die Reduktion, ausgehend von einem Fundamentalsystem $w_{1}(z), \ldots, w_{n}(z)$ der Differentialgleichung $L_{n}(w)=w^{(n)}+a_{n-1}(z) w^{(n-1)}+$ $\ldots+a_{0}(z) w=0$ so oft angewandt, bis eine Differentialgleichung der Form

$$
v^{\prime}(z)=A^{*}(z) v(z)
$$

erreicht wird. Daraus folgt, da $v(z)$ in $|z|<\infty$ meromorph und von endlicher Ordnung ist, für die meromorphe Funktion $A^{*}(z) m\left(r, A^{*}(z)\right)=$ $O(\log r)$. Wiederholte Anwendung des Hilfssatzes ergibt $m\left(r, a_{j}\right)=$ $O(\log r)$ für $j=1, \ldots, n-1$. Mit Hilfe der Differentialgleichung

$L_{n}(w)=0$ erhält man schliesslich noch $m\left(r, a_{0}\right)=O(\log r)$. Mithin gilt der

Satz 1. In der Differentialgleichung

$$
w^{(n)}+a_{n-1}(z) w^{(n-1)}+\ldots+a_{0}(z) w=0
$$

sind die ganzen Koeffizienten genau dann Polynome, wenn jede Lösung der Differentialgleichung von endlicher Ordnung ist.

Resultate, die sich auf den Fall beziehen, dass gewisse der Koeffizienten $a_{j}(z)$ ganze transzendente Funktionen sind, findet man bei M. Frei [3].

2. Bei den folgenden Bemerkungen zu Satz 1 wird durchweg angenommen, dass $L_{m}(w)=0$ eine lineare Differentialgleichung der Ordnung $m$ von der Form (D) mit Polynomkoeffizienten $\left(a_{m}(z) \equiv 1\right)$ ist. Sind $w_{1}(z)$, $\ldots, w_{n}(z)$ ganze Funktionen endlicher Ordnung und ist die Wronskische Determinante $W\left(w_{1}, \ldots, w_{n}\right)$ nullstellenfrei, dann genügen diese Funktionen der Differentialgleichung

$$
W\left(w, w_{1}, \ldots, w_{n}\right)=0,
$$

die sich wegen $W\left(w_{1}, \ldots, w_{n}\right) \neq 0$ auf die Normalform

$$
w^{(n)}+a_{n-1}(z) w^{(n-1)}+\ldots+a_{0}(z) w=0
$$

bringen lässt, wobei die Koeffizienten $a_{j}(z)$ ganze Funktionen sind. Da jede Lösung dieser Differentialgleichung eine endliche Ordnung besitzt, sind die Koeffizienten $a_{j}(z)$ nach Satz 1 Polynome, und die Differentialgleichung ist von der Form $L_{n}(w)=0$. Umgekehrt gilt für jedes Fundamentalsystem von $L_{n}(w)=0 \quad W(z) \neq 0$. Danach bestimmen $n$ ganze Funktionen $w_{1}(z), \ldots, w_{n}(z)$ von endlicher Ordnung genau dann eine Differentialgleichung $L_{n}(w)=0$, wenn die mit diesen Funktionen gebildete Wronskische Determinante nullstellenfrei ist. 
Nach G. Frobenius [4] heisst eine Differentialgleichung $L_{n}(w)=0$ irreduzibel, wenn sie mit keiner Differentialgleichung $L_{m}(w)=0$ der Ordnung $m<n$ eine Lösung gemeinsam hat, sonst reduzibel. Ist $L_{n}(w)=0$ reduzibel, dann gibt es mindestens eine irreduzible Differentialgleichung $L_{v}(w)=0$ der Ordnung $v<n$, deren sämtliche Integrale $L_{n}(w)=0$ genügen [11]. Ist $w_{1}(z), \ldots, w_{v}(z)$ ein Fundamentalsystem von $L_{v}(w)=0$, dann gilt $W\left(w_{1}, \ldots, w_{v}\right) \neq 0$, und für $\bar{v}<v$ dieser Funktionen hat die zugehörige Wronskische Determinante stets Nullstellen. Sonst bestimmen nämlich die $\bar{v}$ Funktionen $w_{v_{1}}(z), \ldots, w_{\nu_{\bar{v}}}(z)$ eine Differentialgleichung $L_{\bar{\nu}}(w)=0$, die mit $L_{\nu}(w)=0$ mindestens eine Lösung gemeinsam hat, was der Irreduzibilität von $L_{v}(w)=0$ widerspricht. Hat $L_{n}(w)=0$ umgekehrt die Eigenschaft, dass schon für $\varrho<n$ Lösungen $w_{1}(z), \ldots$, $w_{\varrho}(z)$ eines Fundamentalsystems die Wronskische Determinante nullstellenfrei ist, dann gibt es eine Differentialgleichung $L_{\varrho}(w)=0$, die mit $L_{n}(w)=0$ die Lösungen $w_{1}(z), \ldots, w_{\varrho}(z)$ gemeinsam hat. $L_{n}(u)=0$ ist also reduzibel. Danach gilt der

Satz 2. Die Differentialgleichung $\quad L_{n}(w)=w^{(n)}+a_{n-1}(z) w^{(n-1)}+\ldots$ $+a_{0}(z) w=0$ mit Polynomkoeffizienten ist genau dann reduzibel, wenn $\varrho, 1 \leqq \varrho<n, \quad$ Lösungen $w_{1}(z), \ldots, w_{\varrho}(z)$ existieren, für welche $W\left(w_{1}, \ldots, w_{\varrho}\right) \neq 0$ gilt.

Hat $L_{n}(w)=0$ eine nullstellenfreie ganze Lösung $w(z)=e^{p(z)}, p(z)$ ein Polynom, dann ist sie wegen $L_{1}(w)=w^{\prime}-p^{\prime}(z) w=0$ reduzibel. Die Differentialgleichung $L_{n}(w)=0$ hat höchstens $(n-1)$ linear unabhängige Polynomlösungen. Wird diese Maximalanzahl von einer Differentialgleichung $L_{n}(w)=0$ erreicht*), dann ist $L_{n}(w)=0$ irreduzibel. Ist nämlich im Gegensatz dazu $L_{n}(w)=0$ reduzibel, dann gibt es eine irreduzible Differentialgleichung $L_{v}(w)=0$ mit einem Fundamentalsystem $w_{1}(z), \ldots, w_{v}(z)$. Darunter kommt eine ganze transzendente Funktion vor. Es sei $w_{1}(z)$ ganz transzendent. Dann sind $w_{2}(z), \ldots, w_{v}(z)$ linear unabhängige Polynome. $L_{n}(w)$ hat eine Zerlegung der Form $L_{n}(w) \equiv$ $L_{n-v}\left(L_{v}(w)\right)$. Für $j=v+1, \ldots, n$ sind die Funktionen $L_{\nu}\left(u_{j}(z)\right)=p_{j}(z)$ nicht identisch verschwindende und linear unabhängige Polynome. Wegen $L_{n}\left(w_{j}\right)=L_{n-v}\left(p_{j}(z)\right)=0$ hätte dann die Differentialgleichung $L_{n-v}(u)=0$ $n-v$ linear unabhängige Polynomlösungen, was nicht möglich ist.

*) Das ist möglich. Die Funktionen $w_{j}(z)=z^{j}, j=1, \ldots, n-1$, genügen einer Differentialgleichung $L_{n-1}(w)=a_{n-1}(z) w^{(n-1)}+\ldots+a_{0}(z) w=0$ mit Polynomkoeffizienten. $L_{n}(w)=w^{(n)}+L_{n-1}(w)=0$ hat die Polynomlösungen $z, z^{2}, \ldots, z^{n-1}$ und eine ganze transzendente Lösung der Ordnung $n$. 
3. In der Differentialgleichung

$$
z^{2} w^{\prime \prime}+z a(z) w^{\prime}+b(z) w=0, b(z) \neq 0,
$$

seien die Koeffizienten $a(z), b(z)$ hclomorph in $G=\{z ; R<|z|<\infty\}$ und rational in $z=\infty$ :

$$
\begin{aligned}
& a(z)=A z^{\alpha}\left(1+O\left(\frac{1}{z}\right)\right) \\
& b(z)=B z^{\beta}\left(1+O\left(\frac{1}{z}\right)\right) .
\end{aligned}
$$

$\alpha$ und $\beta$ sollen den Bedingungen $\alpha \geqq 0, \beta \geqq 0$ und $\alpha+\beta \geqq 1$ genügen. Wir nehmen an, dass $\left(\bar{D}^{\prime}\right)$ eine in $G$ holomorphe Lösung $w(z)$ hat, für die $z=\infty$ wesentliche Singularität ist. Dann ist in der Laurententwicklung $u(z)=g(z)+\gamma\left(\frac{1}{z}\right) g(z)$ eine ganze transzendente Funktion, deren Ordnung $\lambda(g)$ mit der lokalen Ordnung $\lambda(w, \infty)=\varlimsup_{r \rightarrow \infty} \frac{\log \log M(r, w)}{\log r}$ übereinstimmt. $g(z)$ ist in $G$ Lösung der Differentialgleichung

$$
z^{2} y^{\prime \prime}+z a(z) y^{\prime}+b(z) y=h(z)
$$

$h(z)$ ist holomorph in $G$ und rational in $z=\infty$. Es gilt also $\frac{|h(z)|}{M(r, g)} \rightarrow 0$ für $r \rightarrow \infty$. Ist $v(r)=v(r, g)$ der Zentralindex von $g(z)$ und $\zeta$ eine Stelle auf der Kreislinie $|z|=r>R$, an der $|g(\zeta)|=M(r, g)$ ist, dann gilt [13]

$$
\frac{g^{(j)}(\zeta)}{g(\zeta)}=\left(\frac{v(r)}{\zeta}\right)^{j}\left(1+\eta_{j}(\zeta)\right), j=1,2
$$

(4) ist richtig für alle $\zeta$, deren Betrag $r=|\zeta|$ ausserhalb einer $r$-Menge $\Delta$ von endlichem logarithmischen Mass liegt; die Grössen $\eta_{j}(\zeta)$ streben gegen Null, wenn $|\zeta|$ über zulässige $r$ (d.h. $r \notin 4)$ nach $\infty$ geht. Mit (4) geht (3) wegen $\left|\frac{h(\zeta)}{g(\zeta)}\right| \rightarrow 0$ über in

$$
\left(\frac{v(r)}{\zeta}\right)^{2}+A \zeta^{\alpha-1}\left(\frac{v(r)}{\zeta}\right)\left(1+\varepsilon_{1}(\zeta)\right)+B \zeta^{\beta-2}\left(1+\varepsilon_{2}(\zeta)\right)=0
$$

$\varepsilon_{j}(\zeta) \rightarrow 0$ für $|\zeta| \rightarrow \infty$ über zulässige $\zeta$. Da für ganzes transzendentes $g(z)$ der Zentralindex $v(r, g)$ monoton wachsend nach $\infty$ geht bei $r \rightarrow \infty$, interessieren von $\left(3^{\prime}\right)$ nur Lösungen $v(r)$ mit $v(r) \rightarrow \infty$ bei $r \rightarrow \infty$. Aus $\left(3^{\prime}\right)$ folgt 


$$
v(r)=c r^{\sigma}(1+\eta(r)), \quad c \neq 0 \text { eine Konstante und } 0<\sigma<\infty \text {. }
$$

$\left(5^{\prime}\right)$ gilt für alle $r \notin \Delta$, und dort strebt $\eta(r)$ gegen Null bei $r \rightarrow \infty$. Dabei ist

$$
\begin{aligned}
& \sigma=\alpha \geqq 1 \quad \text { für } \beta<\alpha \\
& \sigma=\frac{\beta}{2} \geqq \frac{1}{2} \quad \text { für } \alpha \leqq \frac{\beta}{2} \\
& \sigma=\alpha \geqq 1 \text { oder } \quad \text { für } \frac{\beta}{2}<\alpha \leqq \beta,
\end{aligned}
$$

also $\sigma \geqq \frac{1}{2}$. Nun lässt sich nach einer in der Theorie der Wertverteilung mehrfach benutzten Schlussweise zeigen, dass $\left(5^{\prime}\right)$ für alle hinreichend grossen $r$ gilt. Es sei $\Delta_{\varrho}=\{r ; \varrho \leqq r \in \Delta\}$ und $\varphi(\varrho)=\int_{\Delta_{\varrho}} \frac{d t}{t}$; dabei ist $\varphi(\varrho) \downarrow 0$ für $\varrho \rightarrow \infty$, also $\varphi(\varrho) \leqq \eta<\frac{1}{2}$ für alle $\varrho>R_{0}(\eta)>R$. Weiter gilt für alle zulässigen $r \geqq R_{1}(\eta)$

$$
c r^{\sigma}(1-\eta)<v(r)<c r^{\sigma}(1+\eta) .
$$

Wir betrachten nun ein beliebiges $r \geqq 2 R(\eta)=2 \max \left(R_{0}, R_{1}\right)$ aus der Ausnahmemenge $\Delta$ und setzen mit $\eta<\eta^{\prime}<\frac{1}{2} \quad r_{1}=r e^{\eta^{\prime}}, \quad r_{0}=r e^{-\eta^{\prime}}>$ $2 R(\eta) e^{-1 / 2}>R(\eta)$. Dann gibt es zulässige $r^{\prime}$, die $r_{0}<r^{\prime}<r$ genügen. Im anderen Fall würde $\left\langle r_{0}, r\right\rangle$ zu $\Delta$ gehören, was wegen $\eta^{\prime}=\log \frac{r}{r_{0}} \leqq$ $\varphi\left(r_{0}\right)<\varphi(R(\eta)) \leqq \eta$ nicht möglich ist. Ebenso folgt die Existenz von zulässigen $r^{\prime \prime}$ mit $r<r^{\prime \prime}<r_{1}$. Unter diesen zulässigen $r$-Werten lassen sich zwei Werte $r^{\prime}$ und $r^{\prime \prime}, r^{\prime}<r<r^{\prime \prime}$, so wählen, dass $\log \frac{r^{\prime \prime}}{r^{\prime}}<\eta$ gilt. Nun ist

$$
\frac{v(r)}{r^{\sigma}} \leqq \frac{v\left(r^{\prime \prime}\right)}{r^{\sigma}}=\frac{v\left(r^{\prime \prime}\right)}{r^{\prime \prime}}\left(\frac{r^{\prime \prime}}{r}\right)^{\sigma} \leqq c(1+\eta) e^{\sigma \eta}<c(1+2 \eta)^{\sigma+1}
$$

und

$$
\frac{v(r)}{r^{\sigma}} \geqq \frac{v\left(r^{\prime}\right)}{r^{\sigma}}=\frac{v\left(r^{\prime}\right)}{r^{\prime \sigma}}\left(\frac{r^{\prime}}{r}\right)^{\sigma} \geqq c(1-\eta)\left(\frac{r^{\prime}}{r^{\prime \prime}}\right)^{\sigma} \geqq c(1-2 \eta)^{\sigma+1},
$$


also

$$
c(1-2 \eta)^{\sigma+1} \leqq \frac{v(r)}{r^{\sigma}} \leqq c(1+2 \eta)^{\sigma+1} .
$$

Da $\eta>0$ beliebig war, gilt $\left(5^{\prime}\right)$ mit passendem $\eta(r), \lim _{r \rightarrow \infty} \eta(r)=0$, für alle $r>R$. Damit ist, da aus $\lim _{r \rightarrow \infty} v(r) r^{-\sigma}=c \lim _{r \rightarrow \infty} r^{-\sigma} \log M(r, g)=\frac{c}{\sigma}$ folgt, $\lambda(g)=\lambda(w, \infty)$ bestimmt, nämlich

$$
\lambda(w, \infty)=\sigma \geqq \frac{1}{2} .
$$

4. Wir nehmen jetzt wie in 3. an, dass die Koeffizienten $a(z), b(z)$ in $\left(\mathrm{D}^{\prime}\right)$ holomorph in $G$ sind, machen aber über ihr Verhalten in $z=\infty$ keine weiteren Annahmen. ( $\left.\mathrm{D}^{\prime}\right)$ hat mindestens eine multiplikative Lösung*) $w_{1}(z)=z^{\varrho} 1 u(z)$, wobei $u(z)$ holomorph in $G$ ist. Durch die Transformation $w(z)=z^{\varrho} 1 y(z)$ geht $\left(\mathrm{D}^{\prime}\right)$ über in $z^{2} y^{\prime \prime}+z\left(2 \varrho_{1}+a(z)\right) y^{\prime}+b(z) y=0$. Diese Differentialgleichung hat jetzt eine in $G$ holomorphe Lösung $y(z)=$ $u(z)$. Wir dürfen danach annehmen, dass schon $\left(\mathrm{D}^{\prime}\right)$ eine in $G$ holomorphe Lösung hat. Hat das charakteristische Polynom det $(A-x E)$ der zu einem Fundamentalsystem von $\left(\mathrm{D}^{\prime}\right)$ in $G$ gehörigen Substitutionsmatrix $A$ zwei verschiedene Nullstellen $\varkappa_{1}$ und $\varkappa_{2}$, dann gibt es noch eine zweite multiplikative Lösung $w_{2}(z)=z^{\varrho} v(z), \varrho \neq 0$ und $v(z)$ holomorph in $G$. Im Falle $\varkappa_{1}=\varkappa_{2}$ existiert eine von $w_{1}(z)$ linear unabhängige Lösung $w_{2}(z)$ mit der Eigenschaft, dass $y(z)=\frac{d}{d z} \frac{w_{2}(z)}{w_{1}(z)}$ eine in $G$ meromorphe Funktion ist. $y(z)$ ist Lösung der Differentialgleichung

$$
z y^{\prime}+\left(2 z \frac{u^{\prime}}{u}+a(z)\right) y=0 \text {. }
$$

Danach gilt mit $\frac{-a(z)}{z}=\frac{a_{-1}}{z}+\sum_{\substack{-\infty \\ j \neq-1}}^{\infty} a_{j} z^{j}=\frac{a_{-1}}{z}+\frac{d g}{d z}, g(z)$ holomorph in $G$ :

$$
y(z)=z^{a}-1 \frac{e^{g(z)}}{u^{2}(z)}=\frac{d H}{d z} .
$$

Da $y(z)$ in $G$ eindeutig ist, muss $a_{-1}=v$ eine ganze Zahl sein. $y(z)$ hat Doppelpole an den Nullstellen $\zeta$ von $u(z)$. Weiter gilt Res $y(z)=0$, wie

*) Man vgl. dazu [1]. 
man durch Reihenansatz $u(z)=u^{\prime}(\zeta)(z-\zeta)+\ldots$ unter Benützung von $\zeta u^{\prime \prime}(\zeta)+a(\zeta) u^{\prime}(\zeta)=0$ bestätigt. Mit $\int_{|z|=r} \frac{d H}{d z} d z=2 \pi i c$ erhalten wir in $H(z)-c \log z=F(z)$ eine in $G$ meromorphe Funktion mit einfachen Polstellen an den Nullstellen $\zeta$ von $u(z) . v(z)=u(z) \cdot F(z)$ ist also holomorph in $G$, und $w_{2}(z)$ ist von der Form

$$
w_{2}(z)=c u(z) \log z+v(z) ;
$$

dafür kann auch $w_{2}(z)=c u(z) \log z+z^{\varrho} v^{*}(z)$, $\varrho$ eine ganze Zahl, geschrieben werden, wobei $v^{*}(z)$ wieder in $G$ holomorph ist. $\mathrm{Zu} z=\infty$ gehört also ein kanonisches Fundamentalsystem [1]

(8) $x_{1} \neq x_{2}:$

$$
w_{1}(z)=u(z) \quad w_{1}(z)=u(z)
$$

$$
w_{2}(z)=z^{\varrho} v(z) \quad \varkappa_{1}=\varkappa_{2}: \quad w_{2}(z)=c u(z) \log z+z^{\varrho} v(z) .
$$

Aus der Struktur dieser Lösungen ist zu erkennen, dass das Verhalten von $|w(z)|$ einer beliebigen Lösung $w(z)=c_{1} w_{1}(z)+c_{2} w_{2}(z)$ bei $|z| \rightarrow \infty$ im wesentlichen durch das Anwachsen von $|u(z)|$ und $|v(z)|$ bestimmt wird, wenn $z$ der Bedingung $|\arg z| \leqq \Theta$ unterworfen wird.

Nach Konstruktion von $v(z)$ gilt im Falle $\varkappa_{1}=\varkappa_{2}$ für die lokale Ordnung $\lambda(v, \infty): \lambda(v, \infty) \leqq \max (\lambda(u, \infty), \lambda(F, \infty))$. Weiter gilt, da die lokalen Ordnungen von $F(z)$ und $F^{\prime}(z)$ gleich sind $[14], \lambda(F, \infty)=$ $\lambda\left(F^{\prime}, \infty\right)=\lambda(y, \infty) \leqq \max \left(\lambda(u, \infty), \lambda\left(e^{g}, \infty\right)\right)$, also

$$
\lambda(v, \infty) \leqq \max \left(\lambda(u, \infty), \lambda\left(e^{g}, \infty\right)\right) .
$$

5. Bilden wir mit dem Fundamentalsystem (8) die Wronskische Determinante, dann erhalten wir

$$
W(z)=W\left(w_{1}, w_{2}\right)=z^{\varrho-1}\left(\frac{c u^{2}}{z^{\varrho}}+\varrho u v+z\left(u v^{\prime}-u^{\prime} v\right)\right),
$$

wobei für $c \neq 0 \quad \varrho$ eine ganze Zahl ist. Zusammen mit $\frac{W^{\prime}(z)}{W(z)}=$ $-\frac{a_{-1}}{z}+\frac{d g}{d z}$ folgt, da $z^{1-\varrho} W(z)$ in $G$ eindeutig ist, $z^{v} e^{g(z)}=\frac{c u^{2}}{z^{\varrho}}+\varrho u v+z\left(u v^{\prime}-u^{\prime} v\right), \quad v$ eine ganze Zahl.

Daraus folgt, wenn die lokalen Ordnungen von $u(z)$ und $v(z)$ endlich sind, durch Übergang zu den Schmiegungsfunktionen

$$
\begin{aligned}
& m\left(r, e^{g}\right) \leqq 3 m(r, u)+m(r, v)+c_{1} \log r, \quad c \neq 0 \\
& m\left(r, e^{g}\right) \leqq m(r, u)+m(r, v)+c_{2} \log r, \quad c=0
\end{aligned}
$$


also $m\left(r, e^{g(z)}\right)=O\left(r^{k}\right), 0 \leqq k<\infty$. Das ist nur möglich, wenn $g(z)$ und damit auch $a(z)$ in $z=\infty$ von rationalem Verhalten sind. Aus

$$
b(z)=-z^{2} \frac{w^{\prime \prime}}{w}-z a(z) \frac{w^{\prime}}{w}
$$

folgt mit $w(z)=u(z) \quad m(r, b(z))=O(\log r)$, d.h. $\quad b(z)$ verhält sich ebenfalls rational in $z=\infty$.

Wir nehmen nun umgekehrt an, dass sich die Koeffizienten $a(z)$ und $b(z)$ rational in $z=\infty$ verhalten. Dann sind die Voraussetzungen von 3. erfüllt. Im Fall $c=0$ sind danach die lokalen Ordnungen von $u(z)$ und $v(z)$ endlich. Ist $c \neq 0$, dann liefert 3 . zunächst $\lambda(u, \infty)<\infty$, und zusammen mit (9) folgt wieder $\lambda(v, \infty)<\infty$. Wir setzen $L(\infty)=\lambda(u, \infty)+$ $\lambda(v, \infty)$ und fassen das Ergebnis zusammen in dem

Satz 3. Für eine Differentialgleichung $z^{2} w^{\prime \prime}+z a(z) w^{\prime}+b(z) w=0$ mit in $R<|z|<\infty$ holomorphen Koeffizienten und mit dem kanonischen Fundamentalsystem (8) ist $L(\infty)$ genau dann endlich, wenn sich die Koeffizienten rational in $z=\infty$ verhalten. $z=\infty$ ist genau dann eine Stelle der Bestimmtheit, wenn $L(\infty)=0$ ist.

Nach Definition einer Bestimmtheitsstelle verhalten sich $u(z), v(z)$ rational in $z=\infty$, also $L(\infty)=0$. Ist umgekehrt $L(\infty)=0$, dann muss wegen (10) $\alpha=0$ und nach $3 . u(z)$ rational in $z=\infty$ sein. Das ist aber nur für $\alpha=\beta$ möglich. Wegen $\lim _{z \rightarrow \infty} \frac{a(z)}{z}=\lim _{z \rightarrow \infty} \frac{b(z)}{z}=0$ für $\alpha=$ $\beta=0$ ist $z=\infty$ Stelle der Bestimmtheit. Das gilt erst recht für $\alpha \leqq 0$ und $\beta \leqq 0$.

6. Für die weiteren Betrachtungen ist es nützlich, die Abschätzung (9) zu ergänzen. Ist $c \neq 0$ also $w_{2}(z)=c u(z) \log z+v(z)$, dann genügt $v(z)$ der inhomogenen Differentialgleichung

$$
\begin{aligned}
z^{2} v^{\prime \prime}+z a(z) v^{\prime}+b(z) v & =-c\left(2 z u^{\prime}+(a(z)-1) u\right) \\
& =-c u(z)\left(2 z \frac{u^{\prime}}{u}+a(z)-1\right) \\
& =-c u(z) H(z)
\end{aligned}
$$

Die in $G$ meromorphe Funktion $U(z)=2 z \frac{u^{\prime}}{u}$ genügt mit $\Phi(z)=1-a(z)$ der Differentialgleichung

$$
z U^{\prime}=-2 b(z)+\Phi(z) \cdot U-\frac{U^{2}}{2}
$$


und $H(z)=U(z)-\Phi(z)=2 z \frac{u^{\prime}}{u}+a(z)-1$ ist eine in $G$ meromorphe Lösung der Riccatischen Differentialgleichung

$$
y^{\prime}=\frac{-y^{2}}{2 z}+D(z), \quad D(z)=\frac{\Phi^{2}(z)}{2 z}-\frac{2 b(z)}{z}-\Phi^{\prime}(z) .
$$

Da (12) mindestens eine in $G$ eindeutige Lösung hat, nämlich $y(z)=H(z)$, kann $D(z)$ nicht identisch verschwinden. Dann folgt aber wegen $m(r, D)=$ $O(\log r)$ aus $(12)$

$$
m(r, H)=O(\log r), \quad m\left(r, \frac{1}{H}\right)=O(\log r)
$$

also $m(r, u) \leqq m(r, v)+c \log r, \lambda(u, \infty) \leqq \lambda(v, \infty)$. Danach gilt

$$
\begin{aligned}
& \alpha \leqq \max (\lambda(u, \infty), \lambda(v, \infty)) \text { für beliebiges } c, \\
& \lambda(u, \infty) \leqq \lambda(v, \infty) \leqq \max (\alpha, \lambda(u, \infty)) \text { für } c \neq 0 .
\end{aligned}
$$

Hat $\left(\mathrm{D}^{\prime}\right)$ ein kanonisches Fundamentalsystem $w_{1}(z)=u(z), w_{2}(z)=z^{\varrho} v(z)$ bei $z=\infty$, dann genügt $v(z)$ der Differentialgleichung

$$
z^{2} v^{\prime \prime}+z a^{*}(z) v^{\prime}+b^{*}(z) v=0
$$

mit $a^{*}(z)=a(z)+2 \varrho=A^{*} z^{\alpha^{*}}\left(1+\left(\frac{1}{z}\right)\right), b^{*}(z)=\varrho(\varrho-1)+\varrho a(z)+$ $b(z)=B^{*} z^{\beta^{*}}\left(1+\left(\frac{1}{z}\right)\right)$. Danach ist $x=\alpha^{*}, \beta^{*} \leqq \max (\alpha, \beta)$. Wir erhalten für $\beta<\alpha$

$$
\lambda(u, \infty)=\lambda(v, \infty)=\alpha, L(\infty)=2 \alpha
$$

und für $\quad \alpha \leqq \frac{\beta}{2}$

$$
\lambda(u, \infty)=\lambda(v, \infty)=\frac{\beta}{2}, L(\infty)=\beta .
$$

Ist $\frac{\beta}{2}<\alpha \leqq \beta$, dann sind für $u(z), v(z)$ die Ordnungen $\beta-\alpha$ und $\alpha$ möglich. Wegen (13) ist $\lambda(u, \infty)=\lambda(v, \infty)=\beta-\alpha$ nicht möglich. Wählen wir, wenn $u(z)$ und $v(z)$ verschiedene Ordnungen haben, die Bezeichnung so, dass $\lambda(u, \infty)<\lambda(v, \infty)$ gilt, dann erhalten wir für $\frac{\beta}{2}<\alpha \leqq \beta$

$$
\lambda(u, \infty)=\alpha=\lambda(v, \infty), \quad L(\infty)=2 \alpha
$$

oder

$$
\lambda(u, \infty)=\beta-\alpha<\lambda(v, \infty)=\alpha, L(\infty)=\beta .
$$


Nach (13) gelten diese Beziehungen unverändert auch im Fall $c \neq 0$, so dass wir für $\left(\mathrm{D}^{\prime}\right)$ erhalten

$$
\begin{aligned}
\beta<\alpha: \quad \lambda(u, \infty)=\lambda(v, \infty)=\alpha, \quad L(\infty)=2 \alpha \\
\alpha \leqq \frac{\beta}{2}: \quad \lambda(u, \infty)=\lambda(v, \infty)=\frac{\beta}{2}, \quad L(\infty)=\beta \\
\frac{\beta}{2}<\alpha \leqq \beta: \lambda(u, \infty)=\lambda(v, \infty)=\alpha, \quad L(\infty)=2 \alpha \quad \text { oder } \\
\lambda(u, \infty)=\beta-\alpha<\lambda(v, \infty)=\alpha, \quad L(\infty)=\beta .
\end{aligned}
$$

Wegen der Annahme $\alpha+\beta \geqq 1$, die bedingt, dass $z=\infty$ eine Unbestimmtheitsstelle ist, gilt

$$
L(\infty) \geqq 1
$$

$L(\infty)=1$ ist nur möglich für $L(\infty)=\beta=1$, also nach (14) für $\alpha=0$, $\beta=1$, und für $\alpha=\beta=1$. Dazu gehören die Differentialgleichungen

$$
\begin{gathered}
z^{2} w^{\prime \prime}+z\left(a_{0}+\frac{a_{-1}}{z}+\ldots\right) w^{\prime}+\left(b_{1} z+b_{0}+\frac{b_{-1}}{z}+\ldots\right) \begin{array}{c}
w=0 \\
b_{1} \neq 0
\end{array} \\
z^{2} w^{\prime \prime}+z\left(a_{1} z+a_{0}+\frac{a_{-1}}{z}+\ldots\right) w^{\prime}+ \\
\left(b_{1} z+b_{0}+\frac{b_{-1}}{z}+\ldots\right) w=0, a_{1} b_{1} \neq 0 .
\end{gathered}
$$

Da $\left(\mathrm{D}_{1}\right)$ keine in $z=\infty$ rationale Lösung hat, sind $u(z)$ und $v(z)$ transzendent, so dass nach $(14) \lambda(u, \infty)=\lambda(v, \infty)=\frac{1}{2}, L(\infty)=1$ gilt. Ist für $\left(\mathrm{D}_{2}\right) L(\infty)=1$, dann muss $\lambda(u, \infty)=0$ gelten, also $u(z)$ rational in $z=\infty$ sein. Ist umgekehrt $u(z)$ rational in $\infty$, dann muss, da $z=\infty$ Unbestimmtheitsstelle ist, $v(z)$ transzendent sein. Nach (14) ist dann $\lambda(v, \infty)=\alpha=1$ und somit $L(\infty)=1$.

Wir fassen diese Ergebnisse, die Resultate von H. von Koch und O. Perron [10] verschärfen, zusammen in dem

Satz 4. Ist $z=\infty$ eine Unbestimmtheitsstelle der Differentialgleichung $\left(\mathrm{D}^{\prime}\right)$, dann gilt $L(\infty)=\lambda(u, \infty)+\lambda(v, \infty) \geqq 1$. Das Gleichheitszeichen steht genau dann, wenn $\left(\mathrm{D}^{\prime}\right)$ von der Form $\left(\mathrm{D}_{1}\right)$ oder $\left(\mathrm{D}_{2}\right)$ ist mit einer in $z=\infty$ rationalen Lösung $u(z)$. Für jede andere Differentialgleichung ist $L(\infty) \geqq 2$. 
7. Wir bezeichnen die in den Punkten $z_{1}, z_{2}, \ldots, z_{v}=\infty, \quad v \geqq 1$, punktierte $z$-Ebene mit $G_{v}$ und nehmen an, dass die Koeffizienten $a(z)$, $b(z)$ der Differentialgleichung $\left(\mathrm{D}^{\prime}\right)$ holomorph in $G_{v}$ sind. Zu jeder Stelle $z_{j}$ gehört ein kanonisches Fundamentalsystem mit den in einer Umgebung von $z=z_{j}$ holomorphen Funktionen $u_{j}(z), v_{j}(z)$. Sind $\lambda\left(u_{j}, z_{j}\right), \lambda\left(v_{j}, z_{j}\right)$ ihre lokalen Ordnungen, so ist $L\left(z_{j}\right)=\lambda\left(u_{j}, z_{j}\right)+\lambda\left(v_{j}, z_{j}\right)$ genau dann endlich, wenn $a(z)$ und $b(z)$ rational bei $z_{j}$ sind. Die Koeffizienten von $\left(\mathrm{D}^{\prime}\right)$ sind daher genau dann rational in der vollen $z$-Ebene, wenn $L\left(z_{j}\right)$ für jede Stelle $z_{j}$ endlich ist. $L\left(z_{j}\right)=0, j=1, \ldots, v$, charakterisiert die Differentialgleichungen vom Fuchsschen Typus. Wollen wir über diese Klasse von Differentialgleichungen hinauskommen, dann muss für mindestens eine Stelle $z_{j}$ die Grösse $L\left(z_{j}\right)$ positiv und damit $\geqq 1$ sein. Es liegt nun nahe, nach denjenigen Differentialgleichungen zu fragen, für welche an $\mu$ Stellen, $0 \leqq \mu<v, L\left(z_{j}\right)=0$ und an den restlichen $(v-\mu)$ Stellen $L\left(z_{j}\right)=1$ gilt. Es zeigt sich, dass die Forderung $L\left(z_{j}\right)=1$, die die Stelle $z_{j}$ zu einer möglichst schwachen Unbestimmtheitsstelle macht, zu den am Anfang der Arbeit angekündigten Differentialgleichungen der mathematischen Physik führt. Wir betrachten hier nur einige typische Beispiele bei Beschränkung auf den regulären Fall $\lambda\left(u_{j}, z_{j}\right)=\lambda\left(v_{j}, z_{j}\right)$.

Für $v=1$ sind die Differentialgleichungen von der Form $w^{\prime \prime}+A(z) w^{\prime}$ $+B(z) w=0$ mit ganzen Koeffizienten, die genau dann Polynome sind, wenn $L(\infty)=\lambda\left(w_{1}\right)+\lambda\left(w_{2}\right)$ endlich ist. Ist $\alpha_{0}$ bzw. $\beta_{0}$ der Grad von $A(z)$ bzw. $B(z)$, dann gilt $\alpha=\alpha_{0}+1 \geqq 1, \beta=\beta_{2}+2 \geqq 2$, also $L(\infty) \geqq 2$. Es ist $L(\infty)=2$ genau dann, wenn die Differentialgleichung konstante Koeffizienten hat. Sie verschwinden beide genau dann, wenn $z=\infty$ Bestimmtheitsstelle ist.

Für $v=2$ sei $z_{1}=0, z_{2}=\infty$. Liegt die Eulersch 9 Differentialgleichung vor, dann ist $L(0)=L(\infty)=0$ und umgekehrt. Von den Bedingungen $L(0)=0, L(\infty)=1$ führt letztere nach Satz $4 \mathrm{zu}$

$$
a(z)=a_{0}+\frac{a_{-1}}{z}+\ldots, b(z)=b_{1} z+b_{0}+\frac{b_{-1}}{z}+\ldots, \quad 0<|z|<\infty .
$$

Da $z=0$ Stelle der Bestimmtheit sein soll, ergibt sich daraus die Gleichung

$$
z^{2} w^{\prime \prime}=z a_{0} w^{\prime}+\left(b_{1} z+b_{0}\right) w=0 .
$$

Durch Bedingungen, die an die Wurzeln $\varrho_{j}$ der determinierenden Gleichung $\varrho(\varrho-1)+a_{0} \varrho+b_{0}=0$ für die Stelle $z=0$ gestellt werden, reduziert sich die Zahl der Parameter. So führt die Forderung $\varrho_{1}+\varrho_{2}=0 \mathrm{zu}$ $z^{2} w^{\prime \prime}+z w^{\prime}+\left(b_{1} z-x^{2}\right) w=0$. Diese Differentialgleichung geht, wenn mit passendem $\gamma$ die Variable $z$ durch $\gamma z$ ersetzt wird, über in

$$
z^{2} w^{\prime \prime}+z w^{\prime}-\left(z+x^{2}\right) w=0
$$


die durch Zylinderfunktionen gelöst wird.

Ist $L(0)=L(\infty)=1$, dann gilt zunächst wieder $(15)$. Durch $z=\frac{1}{t}$, $w(z(t))=y(t)$ geht (15) über in

$$
t^{2} \frac{d^{2} y}{d t^{2}}+t\left(2-a\left(\frac{1}{t}\right)\right) \frac{d y}{d t}+b\left(\frac{1}{t}\right) y=0 .
$$

Da wegen $L(0)=1$ bei $t=\infty \quad L=1$ gelten muss, folgt

$$
a_{-1}=a_{-2}=\ldots=0, b_{-2}=b_{-3}=\ldots=0 \text {. }
$$

Damit erhalten wir, wenn wir noch die Transformation $z \rightarrow \gamma z$ anwenden,

$$
z^{2} u^{\prime \prime}+z a_{0} u^{\prime}+\left(b_{0}+b\left(z+\frac{1}{z}\right)\right) w=0 .
$$

Mit $a_{0}=1$ und $z=e^{2 i \zeta}, w(z(\zeta))=W(\zeta)$ geht (17) über in die Mathieusche Differentialgleichung

$$
\frac{d^{2} W}{d \zeta^{2}}+\left(\lambda-2 h^{2} \cos 2 \zeta\right) W=0
$$

Nach (14) führt die Bedingung $L(0)=0, L(\infty)=2$, wenn wir nur den Fall $\lambda(u, \infty)=\lambda(v, \infty)$ berücksichtigen, zu den Differentialgleichungen

$$
\begin{aligned}
& z^{2} w^{\prime \prime}+z\left(A_{0} z+A_{1}\right) w^{\prime}+\left(B_{0} z+B_{1}\right) w=0, \quad A_{0} \neq 0 \\
& z^{2} w^{\prime \prime}+z\left(A_{0} z+A_{1}\right) w^{\prime}+\left(B_{0} z^{2}+B_{1} z+B_{2}\right) w=0, \quad B_{0} \neq 0 .
\end{aligned}
$$

Daraus entstehen, wenn eine Wurzel der determinierenden Gleichung für $z=0 \quad$ zu Null gemacht wird, die Differentialgleichungen

$$
\begin{aligned}
& z w^{\prime \prime}+\left(a_{0} z+a_{1}\right) w^{\prime}+b_{0} w=0, \quad a_{0} \neq 0 \\
& z w^{\prime \prime}+\left(a_{0} z+a_{1}\right) w^{\prime}+\left(b_{0} z+b_{1}\right) w=0, \quad b_{0} \neq 0 .
\end{aligned}
$$

Durch eine Transformation $z \rightarrow \gamma z$ geht $\left(\mathrm{D}_{1}\right)$ in die konfluente hypergeometrische Differentialgleichung:

$$
z w^{\prime \prime}+(A-z) w^{\prime}-B w=0
$$

über. Ist in $w(z)=e^{s z} y(z) \quad s$ eine Wurzel der Gleichung $s^{2}+a_{0} s+b_{0}=0$, dann erhalten wir aus $\left(\mathrm{D}_{2}\right)$

$$
z y^{\prime \prime}+\left(\left(a_{0}+2 s\right) z+a_{1}\right) y^{\prime}+\left(s a_{1}+b_{1}\right) y=0 .
$$

Das ist, wenn die Gleichung $s^{2}+a_{0} s+b_{0}=0$ keine Doppelwurzel hat, eine konfluente hypergeometrische Differentialgleichung. Ist $2 s+a_{0}=0$, dann erhalten wir eine Differentialgleichung der Form (16). Die Bedingung $L(0)=0, \quad L(\infty)=2$ führt also zur konfluenten hypergeometrischen Differentialgleichung. 
8. Wir betrachten jetzt die Differentialgleichung $\left(\mathrm{D}^{\prime}\right)$ für beliebiges $v$ und machen neben

$$
L\left(z_{j}\right)=0, j=1,2, \ldots, v-1, L\left(z_{1}\right)=L(\infty)<\infty
$$

die Annahme: Jede Lösung $w(z)$ von $\left(\mathrm{D}^{\prime}\right)$ ist eindeutig in $G_{v}$. Da die Stellen $z_{1}, \ldots, z_{v-1}$ Stellen der Bestimmtheit sind, ist $w(z)$ in $|z|<\infty$ holomorph bis auf endlich viele Polstellen, die unter den Stellen $z_{1}, \ldots, z_{v-1}$ vorkommen. Für $\left(\mathrm{D}^{\prime}\right)$ kann man schreiben

$$
a_{0}(z) w^{\prime \prime}+a_{1}(z) w^{\prime}+a_{2}(z) u=0 ;
$$

die Koeffizienten $a_{j}(z)$ sind Polynome vom Grad $x_{j} \cdot z_{v}=\infty$ ist genau dann Stelle der Bestimmtheit, wenn $\alpha_{1}-x_{0} \leqq-1$ und $\alpha_{2}-\alpha_{0} \leqq-2$ gilt. Die in $R<|z|<\infty$ holomorphen Funktionen u(z), $v(z)$ aus dem kanonischen Fundamentalsystem bei $z=\infty$ sind wegen der Eindeutigkeit jeder Lösung $w(z)$ selber Lösungen von $\left(\mathrm{D}^{\prime \prime}\right)$. Mit der Normierung $\lambda(u, \infty) \leqq$ $\lambda(v, \infty)$ bedingt $L(\infty)>0$ nach den bisherigen Ergebnissen $\lambda(v, \infty) \geqq \frac{1}{2}$. Wir zeigen, dass die Forderung der Eindeutigkeit $\lambda(v, \infty) \geqq 1$ nach sich zieht. Zunächst folgt aus der Annahme $\lambda(v, \infty)=\frac{1}{2}$, da $u(z)$ und $v(z)$ beide $\left(\mathrm{D}^{\prime \prime}\right)$ genügen und nur eine Lösung der Ordnung $\frac{1}{2}$ möglich ist ([13], S. 67), $\lambda(u, \infty)<\frac{1}{2}$, d.h. $u(z)$ ist rational in $z=\infty$. Das ist nur möglich, wenn von den Zahlen $\alpha_{0}-2, \alpha_{1}-1$ und $\alpha_{2}$ mindestens zwei gleich dem Maximum dieser drei Zahlen sind. Das kann aber, da $\lambda(v, \infty)=\frac{1}{2}$ $x_{1}-\alpha_{0} \leqq-1$ und $x_{2}-\alpha_{0} \leqq-1$ bedingt, nicht zutreffen. Die Annahme $\lambda(v, \infty)=\frac{1}{2}$ führt also zu einem Widerspruch. Nach früheren Ergebnissen ist daher $\lambda(v, \infty) \geqq 1$. Unter den angegebenen Voraussetzungen folgt also aus $L(\infty)>0 \quad \lambda(w) \geqq 1$ für jede Lösung $u(z)$ von $\left(\mathrm{D}^{\prime \prime}\right)$.

Ist $\lambda(w)=1$, dann hat die Wronskideterminante $W(z)=W(u(z) \cdot v(z))$ eine Wachstumsordnung, die höchstens gleich 1 ist. Daraus folgt wegen $\frac{W^{\prime}(z)}{W(z)}=-\frac{a_{1}(z)}{a_{0}(z)}$

Geht man von $\frac{a_{2}(z)}{a_{0}(z)}=-\frac{w^{\prime \prime}(z)}{w(z)}-\frac{a_{1}(z)}{a_{0}(z)} \frac{w^{\prime}(z)}{w(z)}$ mit Hilfe des 
Zentralindex $v(r, v)$ von $v(z)$, der wegen $\lambda(v, \infty)=1 \lim _{r \rightarrow \infty} \frac{v(r, v)}{r}=c \neq$ $0, \infty$ genügt, zu der Gleichung

$$
\frac{a_{2}(\zeta)}{a_{0}(\zeta)}=-\left(\frac{v(r, v)}{\zeta}\right)^{2}\left(1+\varepsilon_{1}(\zeta)\right)-\frac{a_{1}(\zeta)}{a_{0}(\zeta)} \frac{v(r, v)}{\zeta}\left(1+\varepsilon_{2}(\zeta)\right)
$$

über, dann folgt $\left|\frac{a_{2}(\zeta)}{a_{0}(\zeta)}\right| \leqq c$ für $|\zeta| \geqq r_{0}$, also

$$
\alpha_{2}-\alpha_{0} \leqq 0
$$

Nach einem Satz von G. Halphen [6] folgt jetzt, dass $w(z)$ die Gestalt

$$
w(z)=R_{1}(z) e^{s_{1} z}+R_{2}(z) e^{s_{2} z}
$$

hat. $R_{1}(z)$ und $R_{2}(z)$ sind rationale Funktionen. Für $L(\infty)>0$ ist $\left|s_{1}\right|+\left|s_{2}\right|>0$. Umgekehrt bilden zwei linear unabhängige Funktionen $R_{1}(z) e^{s_{1} z}$ und $R_{2}(z) e^{s_{2} z}$ stets ein Fundamentalsystem einer Differentialgleichung $\left(\mathrm{D}^{\prime \prime}\right)$, wobei für die Grade $\alpha_{j}$ der Polynome $a(z)$ die Beziehungen

$$
\alpha_{0} \geqq \alpha_{1} \text { und } \alpha_{0} \geqq \alpha_{2}
$$

gelten.

Technische Hochschule Karlsruhe 


\section{Literatur}

[1] Bieberbach, L.: Theorie der gewöhnlichen Differentialgleichungen. - SpringerVerlag (1964).

[2] Сh. Briot - J. C. Bouquet: Mémoire sur l'intégration des équations différentielles au moyen des fonctions elliptiques. - Journ. de l'Ec.pol. 21 (cah 36) (1856).

[3] Frer, M.: Über die Lösungen linearer Differentialgleichungen mit ganzen Funktionen als Koeffizienten. - Comment. Math. Helv. 35 (1961).

[4] Frobenius, G.: Über den Begriff der Irreductibilität in der Theorie der linearen Differentialgleichungen. - Crelles Journal 76 (1873).

[5] Fuchs, L.: Gesammelte Werke Bd. 1 (1904).

[6] Halphen, G.: Sur une nouvelle classe d'équations différentielles linéaires intégrables. - C. R. Acad. Sci. Paris 101 (1885).

[7] Hayman, W. K.: Meromorphic functions. - Oxford (1964).

[8] Malmquist, J.: Sur les fonctions à une nombre fini des branches définies par les équations différentielles du premiere ordre. - Acta math. 36 (1913).

[9] Nevandinsa, R.: Eindeutige analytische Funktionen. - Springer-Verlag (1953).

[10] Perrox, O.: Über einen Satz des Herrn Helge von Koch über die Integrale linearer Differentialgleichungen. - Math. Z. 3 (1919).

[11] Schlesinger, L.: Handbuch der Theorie der linearen Differentialgleichungen. Bd. 1 (1895).

[12] Wruas, A.: Über den Zusammenhang zwischen dem Maximalbetrage einer analytischen Funktion und dem grössten Glied der zugehörigen Taylorschen Reihe. - Acta math. 37 (1914).

[13] Wrттісн, H.: Neuere Untersuchungen über eindeutige analytische Funktionen. Springer-Verlag: (1955).

[14] -»-: Über die Ordnung meromorpher Funktionen. - Arch. Nath. XVI (1965). 